Nouvelles perspectives en sciences sociales

Revue internationale de systémique complexe et d'études relationnelles

Sortir la violence de sa vie : itinéraires d'hommes en

changement, Pierre Turcotte, Québec, Presses de l'Université Laval, coll. « Travail social ", 2012

\title{
Simon Lapierre
}

Volume 9, numéro 1, novembre 2013

URI : https://id.erudit.org/iderudit/1024049ar

DOI : https://doi.org/10.7202/1024049ar

Aller au sommaire du numéro

Éditeur(s)

Prise de parole

ISSN

1712-8307 (imprimé)

1918-7475 (numérique)

Découvrir la revue

Citer ce compte rendu

Lapierre, S. (2013). Compte rendu de [Sortir la violence de sa vie : itinéraires d'hommes en changement, Pierre Turcotte, Québec, Presses de l'Université

Laval, coll. « Travail social ", 2012]. Nouvelles perspectives en sciences sociales, 9(1), 353-356. https://doi.org/10.7202/1024049ar d'utilisation que vous pouvez consulter en ligne. 


\section{Sortir la violence de sa vie : itinéraires d'hommes en changement}

Pierre Turcotte, Québec, Presses de l'Université Laval, coll. «Travail social », 2012.

PAR Simon LAPIERRE

Université d'Ottawa

D ans son livre intitulé Sortir la violence de sa vie : itinéraires d'hommes en changement, Pierre Turcotte traite de l'intervention sociale auprès d'hommes qui ont des comportements violents à l'endroit de leur conjointe. L'auteur s'appuie sur les résultats de ses recherches empiriques et sur ses réflexions personnelles et professionnelles dans le but de contribuer à l'avancement des connaissances théoriques et cliniques sur l'intervention auprès des hommes, particulièrement en contexte de violence conjugale. Plus précisément, ce livre donne la parole à des hommes qui ont entrepris une démarche d'aide en lien avec leur problème de violence, de manière à illustrer leur expérience thérapeutique et leur itinéraire de changement.

Il existe, depuis quelques décennies, plusieurs programmes d'intervention pour conjoints violents - qui ont maintenant tendance à privilégier une approche globale, incluant la santé des hommes, la paternité, etc. - et les chercheurs dans ce domaine se sont surtout intéressés à l'efficacité de ces programmes et aux facteurs facilitant ou entravant la démarche de changement. Ce livre est donc novateur dans la mesure où il donne la parole à des hommes qui ont entrepris une démarche d'aide et s'intéresse à leur expérience thérapeutique.

Il semble important de souligner que l'auteur a fait explicitement le choix de ne pas s'attarder aux considérations théoriques et méthodologiques sous-jacentes à ses travaux : « afin de faciliter la lecture, nous ne présenterons pas ici les considérations théoriques ou méthodologiques de la recherche à l'origine de la 
présente publication " (p. 11). Cela constitue, à mon avis, une importante limite de l'ouvrage. En effet, des informations supplémentaires concernant la méthodologie de l'étude auraient permis d'apprécier davantage la portée des résultats présentés. De plus, considérant que la violence est un sujet qui suscite d'importants débats idéologiques et politiques, opposant les féministes (ou proféministes) et les masculinistes (ou antiféministes), l'absence d'un solide cadre théorique semble particulièrement problématique. Ainsi, certains lecteurs pourraient déceler une tendance masculiniste dans les propos de l'auteur, notamment lorsqu'il met l'accent sur le « coût à payer » pour les hommes qui doivent intégrer les normes de la masculinité sans mentionner les privilèges masculins. L'auteur laisse pourtant entrevoir, à plusieurs endroits, une perspective qui relève davantage de la posture pro-féministe. C'est le cas, par exemple, lorsqu'il soutient que la violence conjugale s'inscrit dans des rapports de pouvoir et de domination sexiste.

De manière plus générale, le livre est divisé en six chapitres. Faisant suite à une courte introduction, le premier chapitre traite sommairement de l'évolution, dans le contexte québécois, de l'intervention auprès des hommes qui ont des comportements violents. Il aborde, notamment, la construction de la violence conjugale comme un problème social qui s'est traduit par la mise en place de services de protection pour les femmes victimes et des services de correction pour les hommes agresseurs. Dans la deuxième partie de ce chapitre, l'auteur définit les principaux concepts utilisés dans ce livre, soit la violence conjugale, les programmes de thérapie, l'expérience thérapeutique, l'itinéraire du changement et le changement.

Les trois chapitres suivants se penchent sur l'expérience des hommes aux différentes étapes de la démarche d'aide. Ainsi, le deuxième chapitre s'intéresse aux motifs de consultation, tels que perçus par les hommes au début de la démarche. À cet égard, l'auteur souligne que, même si les hommes qui ont des comportements violents consultent souvent pour des raisons en lien avec leur relation conjugale, ils ont souvent une attitude de déni face 
au problème : «nos résultats indiquent que seulement le tiers des participants reconnaissent au départ leur responsabilité face à la violence " (p. 20). L'auteur mentionne également que la demande d'aide peut être une épreuve honteuse pour les hommes et met en évidence les coûts affectifs liés à la prise de conscience de la violence (isolement, dépression, pensées suicidaires, etc.). Le troisième chapitre s'intéresse à la qualité de l'accueil reçu, qui constitue un facteur déterminant dans la reconnaissance du problème : « les participants à la présente recherche ont largement témoigné de l'importance de la qualité de la relation interpersonnelle avec l'intervenant, tant du point de vue de la perception d'eux-mêmes que de la modification de leur perception du problème " (p. 69). Le chapitre suivant se penche sur le processus de prise de conscience à l'étape de la thérapie de groupe, mettant en lumière les changements qui peuvent se produire au cours de la thérapie.

Dans le cinquième chapitre, l'auteur se penche plutôt sur l'itinéraire de changement. Il propose un itinéraire de changement en en fonction des trois étapes suivantes : passer du déni à l'approbation du problème; la mise en application de la décision de ne plus réagir par la violence; et développer un meilleur rapport à soi et aux autres. L'auteur insiste également sur l'importance d'aller au-delà de l'arrêt des comportements violents (changement comportemental) pour favoriser un changement plus profond au plan des relations de pouvoir entre les hommes et les femmes.

Dans l'ensemble, le contenu de ces quatre chapitres permet de mieux comprendre l'expérience thérapeutique et l'itinéraire de changement à partir du point de vue d'hommes qui ont entrepris une démarche d'aide. Les propos des participants sont présentés de manière structurée, sans jugement de la part de l'auteur.

Finalement, le sixième chapitre propose certaines pistes concrètes pour l'intervention sociale auprès des hommes qui ont des comportements violents, incluant : encourager les hommes à exprimer leurs émotions; établir rapidement la différence entre émotion et comportement; développer une attitude d'ouverture 
dès l'accueil; accueillir les émotions, mais d'abord centrer l'homme sur l'arrêt d'agir et la reconnaissance de ce qu'est la violence; favoriser la construction d'une solide alliance thérapeutique; favoriser l'aide mutuelle au sein du groupe de traitement; écouter les hommes et les encourager à s'ouvrir à ce qu'ils vivent; voir la démarche thérapeutique des hommes comme une quête de sens; et déconstruire socialement l'étiquette d' " homme violent ». L'auteur aborde également, dans ce chapitre, l'enjeu de l'intervention familiale dans un contexte de violence conjugale, une approche particulièrement populaire aux États-Unis. À cet égard, son point de vue est clair et intéressant :

Cette approche ne tient pas compte en effet des rapports sociaux de sexe et s'appuie sur une conception erronée du genre, se limitant à inclure la violence des femmes dans la lecture de la violence familiale. Il s'agit à notre avis d'une mauvaise lecture sociologique de la violence conjugale issue d'une insuffisance théorique du concept de genre (p. 139).

Ainsi, l'auteur souligne plutôt la nécessité d'une intervention sociale orientée vers un changement personnel et social, qui remet en question des formes plus traditionnelles de masculinité et qui favorise des rapports sociaux plus égalitaires entre les hommes et les femmes.

En somme, même si la contribution théorique de ce livre n'est pas aussi importante qu'elle aurait pu l'être si l'auteur avait exposé clairement son cadre théorique et sa posture idéologique et politique, il n'en demeure pas moins une contribution importante sur le plan clinique. En donnant ainsi la parole à des hommes pour qu'ils puissent s'exprimer sur la démarche d'aide qu'ils ont entreprise, ce livre constitue un outil précieux pour les professionnels ou les futurs professionnels qui souhaitent intervenir auprès d'hommes qui ont des comportements violents. 